\title{
In-vivo-Druckmessungen zum Vergleich medizinischer adaptiver Kompressionssysteme mit maßangefertigter Flachstrick- Kompressionsversorgung
}

\section{Sub-compression interface pressure measurements in vivo, comparing adjustable compression wraps and custom-made flat knit compression stockings}

Autoren

Anett Reißhauer ${ }^{1}$, Simone Kornappel ${ }^{1}$, Emmanouil Tsatrafilis ${ }^{1}$, Andrea Stroux $^{2}$, Max Liebl ${ }^{1}$

Institute

1 Physikalische Medizin und Rehabilitation, Charité-

Universitätsmedizin Berlin, corporate member of Freie Universität Berlin, Humboldt-Universität zu Berlin, and Berlin Institute of Health

2 Institut für Biometrie und Klinische Epidemiologie, CharitéUniversitätsmedizin Berlin, corporate member of Freie Universität Berlin, Humboldt-Universität zu Berlin, and Berlin Institute of Health

Schlüsselwörter

Lymphödem, Phlebo-Lymphödem, medizinische adaptive

Kompressionssysteme, Klettverschluss-Bandagen,

Flachstrick, Kompressionstherapie, In-vivo-Druckmessungen

Key Words

lymphoedema, phlebolymphedema, adjustable compression wrap, compression therapy, flat knit compression, sub-compression interface pressure

online publiziert 08.07 .2020

Bibliografie

Phlebologie 2020; 49: 313-319

DOI 10.1055/a-1134-2021

ISSN 0939-978X

(C) 2020. Thieme. All rights reserved.

Georg Thieme Verlag KG, Rüdigerstraße 14,

70469 Stuttgart, Germany

Korrespondenzadresse

Dr. med. Max Emanuel Liebl, MaHM

Oberarzt, Physikalische Medizin und Rehabilitation

Charité-Universitätsmedizin Berlin, Charitéplatz 1,

10117 Berlin

Tel.: ++ 49/30/450517082

Fax: $++49 / 30 / 450517907$

max.liebl@charite.de

\section{ZUSAMMENFASSUNG}

Hintergrund Die in der Erhaltungsphase der komplexen physikalischen Entstauungstherapie notwendige Kompressionstherapie kann anstelle von Flachstrick-Kompressionsstrümpfen (FS) auch mit vom Patienten selbst anzulegenden Bandagen erfolgen, den sogenannten medizinischen adaptiven Kompressionssystemen (MAK).

Methoden MAK und FS wurden mittels In-vivo-Druckmessungen unter der von Probanden selbst angelegten Kompression direkt verglichen. Dazu wurden Drücke über PicopressMessgeräte und Sensoren (Microlab Elettronica, Italien) an $\mathrm{n}=30$ Patienten mit beidseitigen, symmetrischen, lymphostatischen Ödemen der Unterschenkel erfasst. FS und MAK wurden seitenrandomisiert zugewiesen. Nach Standardeinweisung und initialen Druckmessungen beider Systeme wurden MAK im Zeitverlauf von 2 und $4 \mathrm{~h}$ Tragedauer erneut gemessen. Static-Stiffness-Index (SSI) sowie Druckgradienten der Messhöhen B1-C wurden berechnet.

Ergebnisse MAK zeigen Ruhedruckwerte und SSI im therapeutischen Bereich, die signifikant höher ausfallen als bei FS $(p<0,01$ bzw. $p<0,001)$. MAK erreichen signifikant höhere maximale Arbeitsdrücke $(p<0,001)$. Die Ruhedruckwerte von MAK zeigen nach 2 und $4 \mathrm{~h}$, ohne Nachjustieren, keinen relevanten Druckabfall. Die mittleren Druckgradienten, bezogen auf die Messhöhen B1-C, unterscheiden sich nicht signifikant zwischen den Methoden.

Diskussion Die mit MAK erreichten Druckparameter untermauern deren therapeutische Effektivität. Die Anwendung von MAK ist auch ohne Nachjustieren druckstabil. Die Anwendung (Selbstanlage) kann als therapeutisch wirksam eingestuft werden. Eine gute Einweisung der Patienten ist jedoch bedeutsam.

\section{ABSTRACT}

Background Adjustable Compression Wraps (ACW) are being used as an alternative to flat-knitted compression stockings (CS) in the maintenance phase of complex decongestive therapy treating lymphoedema.

Methods Self-applied ACW and custom-made CS were compared using sub-compression interface pressure measure- 
ments in vivo. Measurements were recorded using manometer-based Picopress-devices in a sample of $n=30$ probands with bilateral symmetric lymphostatic lower leg edema. Legs were randomized in CS side and ACW side. Following a standardized instruction and initial measurements determining pressure for both systems, ACW pressure measurements were repeated after 2 and 4 hours. Static Stiffness Index and pressure gradients between measuring points B1-C were calculated.

Results ACW showed resting pressures and SSI in therapeutic ranges and significantly higher than $C S(p<.01 ; p<.001)$.
$A C W$ reached significantly higher working pressures $(p<.001)$. Resting pressure sub-ACW did not show significant pressure drops after 2 and 4 hours, without re-adjusting. Average pressure gradients between ACW and CS were not differing significantly.

Discussion The pressure values reached with ACW underline their therapeutic effects. Pressures under self-applied ACW are relatively stable, even without re-adjusting. Self-application is interpreted as effective. A thorough instruction of patients is essential.

\section{Hintergrund}

In der Therapie lymphostatischer Ödeme gilt die komplexe physikalische Entstauungstherapie (KPE) als Standard [1]. In der Erhaltungsphase der Therapie wird die Kompressionstherapie hierzulande meist mit maßangefertigten Kompressionsstrümpfen in Flachstrickqualität mit dem Ziel des Volumenerhalts durchgeführt [1]. Die klinische Praxis zeigt, dass es Versorgungssituationen gibt, die den Einsatz der maßangefertigten Kompressionsstrümpfe limitieren. Zu nennen sind hierbei im Speziellen ausgeprägte Hand- und Fingergelenksarthrosen, allgemeine Kraftminderung oder auch die parallel bestehende Notwendigkeit von Wundversorgungen im Ödemgebiet. Alternative Kompressionsbandagen, die mit Klettverschlüssen eine regelmäßige Anpassung des Kompressionsdrucks erlauben, sogenannte medizinische adaptive Kompressionssysteme (MAK), können in der KPE-Phase I und auch in der KPE-Phase II eingesetzt werden [2-7]. Vorteile werden neben der effektiven Entstauung auch für das Selbstmanagement der Kompressionstherapie, die Therapietreue und auch die leichtere Handhabung beschrieben [2, 3]. Insgesamt ist die Studienlage noch spärlich, noch sind nicht alle möglichen Parameter untersucht. MAK scheinen jedoch durch die Möglichkeit der Nachjustierung des Kompressionsdrucks indikationsspezifische Vorteile durch das Ausgleichen von Druckverlusten und eine geringere Fehleranfälligkeit zu haben [2, 3, 5-8].

In einer durchgeführten vergleichenden Auswertung der Differenzen der Volumeneffekte zeigten sich MAK auch ohne regelmäBiges Nachjustieren der Referenztherapie mit FS in der KPE-Phase II nicht unterlegen. Im Vergleich mit FS berichteten Patienten zudem ein signifikant leichteres An- und Ablegen der MAK bei vergleichbarem Tragekomfort [7]. Die AWMF-S2k-Leitlinie „Medizinische Kompressionstherapie der Extremitäten mit medizinischem Kompressionsstrumpf (MKS), phlebologischem Kompressionsverband (PKV) und medizinischen adaptiven Kompressionssystemen (MAK)“ führt MAK inzwischen gar im Leitlinientitel [9].

Die vorliegende Studie untersucht die Selbstanwendung von MAK im Vergleich mit maßangefertigten Flachstrick-Kompressionstrümpfen durch In-vivo-Druckmessungen an einer Stichprobe von Patienten mit symmetrischen Ödemen mit lymphostatischer Komponente in der Erhaltungsphase der KPE. Es sollen Aussagen zu Materialeigenschaften, RAL-Konformität sowie der klinischen Effektivität der MAK im Sinne therapieadäquater und stabiler
Kompressionsdruckwerte in Selbstanlage getroffen werden. Ferner soll beurteilt werden, ob eine sichere Handhabung der MAK in Selbstanlage gegeben ist.

\section{Material und Methoden}

\section{Studiendesign, Rekrutierung und Vorbereitung}

Es wurden In-vivo-Druckmessungen an beidseitigen, symmetrischen, lymphostatischen Unterschenkelödemen geplant, um die Materialeigenschaften, die therapeutische Wirksamkeit und die sichere Handhabung der Selbstanwendung der MAK beurteilen zu können.

Dabei erfolgte eine Randomisierung mit der Anlage von medizinischen adaptiven Kompressionssystemen (MAK) auf der Interventionsseite und der Anlage von maßgefertigten Kompressionsstrümpfen in Flachstrickqualität (FS) auf der Referenzseite. Die Anlage der Kompressionsmittel erfolgte, per Einschlusskriterium, durch die Probanden selbst. Die Stichprobe bestand aus den Teilnehmern einer randomisiert kontrollierten Studie mit dem primären Endpunkt des Ödemvolumens [7]. Die Fallzahl wurde anhand dieses Endpunktes mit $\mathrm{n}=30$ berechnet. Die In-vivo-Druckmessungen wurden vor Beginn der RCT an allen Studienteilnehmern durchgeführt. Die Ergebnisse dieser Untersuchung, das Ödemvolumen betreffend, wurden an anderer Stelle berichtet [7].

Für die Studie wurden Patienten über eine Lymphödem-Spezialambulanz rekrutiert. Eingeschlossen wurden volljährige Patienten mit bilateralem Lymphödem oder phlebolymphostatischem Ödem im Stadium II und einem symmetrischen klinischen Befund ( $\pm 200 \mathrm{ml}$ im Unterschenkelbereich, optoelektronisch gemessen mittels Perometer Typ 400 NT, Software PeroPlus 2000, Pero-System Messgeräte $\mathrm{GmbH}$, Deutschland) mit beidseits positivem Stemmerschem Zeichen. Darüber hinaus mussten sich die Patienten in der Erhaltungsphase der komplexen physikalischen Entstauungstherapie befinden. Ausschlusskriterien waren das Überschreiten der Maximalmaße für die Anwendung der MAK, Hautfalten mit der Notwendigkeit zusätzlicher Polsterung oder Pelottierung sowie eine unklare Ödemgenese. Unverträglichkeit der Kompressionstherapie und Druckschädigungen, auch durch die Messgeräte, wurden a priori als Abbruchkriterien definiert [7].

Nach dem informed consent erhielten die Probanden eine Neuversorgung mit einem maßgefertigten Kompressionsstrumpf 
in Flachstrickqualität in der ihnen auch schon zuvor ärztlich verordneten Kompressionsklasse II (Modell: Flebovar 2, Varitex BV, Niederlande). Für die Interventionsseite wurde ein mehrteiliges MAK (Modell: ReadyWrap, Lohmann \& Rauscher GmbH \& Co KG, Rengsdorf, Deutschland) nach der Konfektionsgrößentabelle des Herstellers angepasst. Entsprechend der Gebrauchsanweisung der MAK wurden die Probanden in die Selbstanlage des Kompressionssystems eingewiesen. Im Rahmen der Einweisung erfolgte eine Überprüfung der Applikation und gegebenenfalls die Korrektur der Anlagetechnik [7].

\section{Druckmessungen}

- Tab. 1 zeigt die vorbereitenden Maßnahmen und das Messprotokoll der In-vivo-Druckmessungen. Als Messpunkte wurden die Höhen B1 (am Übergang von der Achillessehne zum Muskelbauch des M. gastrocnemius, $12 \mathrm{~cm}$ proximal des Malleolus lateralis) und C (Höhe der größten Zirkumferenz der Wade) verwendet [10]. Die Messungen wurden mittels zweier Picopress-Messgeräte (Microlab Elettronica, Italien) und angeschlossener Laptops durchgeführt $[11,12]$. A Abb. 1 illustriert die Platzierung der entsprechenden Picopress-Sensoren.

Zunächst wurden die mittleren Ruhedruckwerte bei B1 und C bestimmt und die Mittelwerte verglichen. Die Druckwerte bei B1 wurden entsprechend der in Deutschland gültigen Klassifizierung der Kompressionsklassen zugeordnet, um die Effektivität der Selbstanlage der MAK durch die Probanden hinsichtlich der aufgebrachten Kompressionsdrücke zu kontrollieren [11, 12].

Aus den mittleren Druckmessungen der supine und standing Position wurde der Static-Stiffness-Index (SSI) berechnet (Differenz standing minus supine bei Messhöhe B1), der der sogenannten „absolute resting pressure difference” (aRPD) entspricht und als wichtiges Maß für die therapeutische Wirksamkeit der Entstauung gilt [11]. Der mittlere SSI der MAK-Seite wurde mit den SSIWerten der Kontrollseite verglichen.

Als weiterer Hinweis für eine wirksame und sichere Handhabung der Selbstanlage wurde der Druckgradient bestimmt. Hierzu wurde aus den Druckwerten an den Messhöhen B1 und C jeweils unter FS und selbstangelegten MAK die Differenz gebildet. Die mittleren Gradienten der MAK-Seite und der FS-Seite wurden verglichen.

Der maximale Arbeitsdruck (exercise) unter beiden Kompressionsvarianten wurde bestimmt, indem die jeweiligen Maximaldruckwerte aus 10 Druckspitzen bei Bewegung (repetitive Dorsalextension und Plantarflexion) gemittelt wurden. Der mittlere maximale Arbeitsdruck der MAK wurde mit dem der FS-Kompression verglichen.

Sodann wurden die Druckmessungen der MAK-Seite nach 2 und 4 Stunden wiederholt, um die Effektivität der nicht nachjustierten MAK im Zeitverlauf überprüfen zu können.

\section{Statistische Methoden}

Für die Randomisierung wurde die PASS-Software (NCSS LLC, Utah, USA) verwendet. Die Zielvariablen wurden, abhängig von ihren Skalierungs- und Verteilungseigenschaften, deskriptiv mittels absoluter und relativer Häufigkeiten für kategoriellen bzw. Mittelwert, Standardabweichung und Range für quantitative Merkmale
D Tab. 1 Protokoll Druckmessungen.

\section{Druckmessungen}

\section{Vorbereitung}

Rekrutierung und informed consent

randomisierte Seitenzuordnung

Abmessen und Bestellen des Kompressionsmaterials

Passformprüfung

Schulung

standardisierte Einweisung der Probanden nach Protokoll bzw. Hersteller-Gebrauchsanweisung einschließlich Probeanlage, ggf. Wiederholungen

Versuchsaufbau

Anlage der Picopress-Sensoren an den Punkten B1 und C beidseits

Messprotokoll

Anlage MAK und Flachstrick durch den Probanden

Einrichten Druckmessgeräte für B1 und C, Nulleichung

Positionierung liegend, Ferse unterlagert, Unterschenkel kontaktlos, 2 min Ruhezeit

supine: Messung liegend an B1 und C ( 30 s stabiler Messwert) exercise: 10-mal repetitive Dorsalextension/Plantarflexion, Messung der Maximaldrücke an B1 und C

Positionierung stehend (hüftbreiter Stand, 2 min ruhiges Stehen) standing: Messung stehend an B1 und C ( 30 s stabiler Messwert) Entfernung des Materials und Neuanlage durch den Probanden

2-mal Wiederholung der Messreihe

Anbehalten des Kompressionsmaterials und der Messsensoren unter den MAK

nach 2h: Messung an B1 und C (Positionierung liegend, Unterschenkel kontaktlos, 2 min Ruhezeit; nur MAK)

nach 4h: Messung an B1 und C (Positionierung liegend, Unterschenkel kontaktlos, 2 min Ruhezeit; nur MAK)

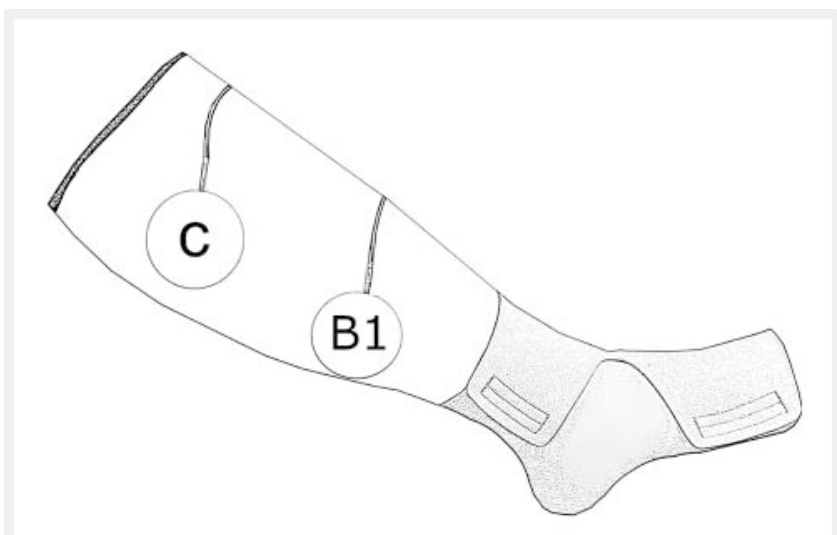

- Abb. 1 Sensoranlage an den Messpunkten B1 und C (hier mit angelegtem Fußteil des MAK). 
analysiert. Vergleiche zwischen den untersuchten Körperseiten wurden mittels t-Tests, bei asymmetrischer Verteilung mittels Wilcoxon-Tests für abhängige Stichproben durchgeführt. Resultierende $\mathrm{p}$-Werte werden als explorativ betrachtet, eine Bonferroni-Korrektur wurde nicht durchgeführt. Die Analysen erfolgten mit SPSS 25.

Die Studie wurde von der Ethikkommission der Charité-Universitätsmedizin Berlin (Ethik-Votum EA1/013/19) genehmigt [7]. Das Datenschutzkonzept wurde zuvor durch den behördlichen Datenschutzbeauftragten beraten.

\section{Ergebnisse}

Die Druckmessungen wurden an $n=30$ Probanden durchgeführt. Die demografischen und krankheitsbezogenen Basisdaten werden in $>$ Tab. 2 dargestellt.

\section{Ruhe- und Arbeitsdruck}

Die mittleren Ruhedruckwerte (liegend) bei B1 betrugen auf der FS-Seite 27,9 $\pm 4,0 \mathrm{mmHg}$, auf der MAK-Seite $33,8 \pm 7,7 \mathrm{mmHg}$. Am Messpunkt $C$ wurden für FS 24,0 $\pm 3,5 \mathrm{mmHg}$ und für MAK $31,1 \pm 6,9 \mathrm{mmHg}$ gemessen. Im Gruppenvergleich zeigten MAK dabei signifikant höhere Druckwerte als die FS-Versorgung der jeweiligen Kontrollseiten (Messhöhe B1: $p=0,002$; Messhöhe C: $\mathrm{p}<0,001)$.

Ordnet man die In-vivo-Drücke bei B1 den deutschen Kompressionsklassen zu, wurden im Liegen bei $97 \%$ der FlachstrickSeite effektiv die KKL II oder III erreicht. Im Bereich der MAK-Seite erreichten $43 \%$ eine KKL II und $43 \%$ ein KKL III nach Selbstanlage, lediglich in 2 Fällen (7\%) wurden Druckwerte angezeigt, die einer KKL I entsprächen, 7 \% erreichten sogar eine KKL IV.

Die Arbeitsdruckwerte (Druckmessungen im Stehen) fielen höher aus. Die Zuordnung zu Kompressionsklassen ergab hier für FS 2-mal die KKL I (7\%), in 28 Fällen ergab sich KKL II (60\%) oder III (33\%). Für die MAK-Seite waren es durchgehend KKL II (23\%), KKL III (50\%) oder gar die KKL IV (27\%) ( A Abb. 2).

\section{Static-Stiffness-Index}

Der Static-Stiffness-Index (SSI) wurde in vivo für MAK und FS berechnet und verglichen. Der gemittelte SSI der MAK zeigte mit $8,2 \pm 6,0$ eine signifikante Überlegenheit der MAK gegenüber FS $(p<0,001)$. Der SSI der FS-Seite fiel mit 2,6 3,5 hier deutlich geringer aus ( $\triangleright$ Abb. 3 ).

\section{Druckgradient}

Der Druckgradient zwischen den Messhöhen B1 und C betrug bei FS 3,9 $( \pm 3,9) \mathrm{mmHg}$ und bei MAK 2,7 $( \pm 4,8) \mathrm{mmHg}$. Im Vergleich der Gradienten zeigte sich kein signifikanter Unterschied $(p=0,26)$.

$73 \%$ der Selbstanlagen von MAK und $83 \%$ der FS-Messungen zeigten in vivo RAL-konforme Druckgradienten [10].

\section{Maximaler Arbeitsdruck (exercise)}

Der gemittelte maximale Arbeitsdruck der FS-Seite wurde mit 32,2 $\pm 4,50 \mathrm{mmHg}$ ermittelt, auf der MAK-Seite mit 45,6 $\pm 10,7 \mathrm{mmHg}$.
- Tab. 2 Demografische Basisdaten und klinische Angaben [7, 13].

\begin{tabular}{|l|l|}
\hline $\mathrm{n}$ & 30 \\
\hline Geschlecht (weiblich) & $25 ; 83,33 \%$ \\
\hline Alter (Jahre) & $57,9 \pm 13,6(21-81)$ \\
\hline Erkrankungsdauer (Jahre) & $23,1 \pm 13,91(2-52)$ \\
\hline Stadium II & $30 ; 100 \%$ \\
\hline BMI (MW; SD; min-max) & $31,9 \pm 7,1(19,7-47,5)$ \\
\hline Adipositas (BMI $\geq 30)$ & $14 ; 46,67 \%$ \\
\hline Adipositas III (BMI $\geq 40)$ & $3 ; 10 \%$ \\
\hline primäres Lymphödem & $6 ; 20 \%$ \\
\hline sekundäres Lymphödem ohne CVI & $8 ; 26,67 \%$ \\
\hline phlebolymphostatisches Ödem & $16 ; 53,33 \%$ \\
\hline CVI mit Adipositas & $8 ; 26,67 \%$ \\
\hline
\end{tabular}

Damit ist bei MAK der gemittelte maximale Arbeitsdruck deutlich höher und signifikant überlegen $(p<0,001)$ ( $\triangleright$ Abb. 4).

\section{Druckmessungen im Zeitverlauf}

Der unter den MAK gemessene zeitliche Druckverlauf zeigte sich stabil. Die mittleren Ruhedruckwerte sanken von $33,8 \mathrm{mmHg}$ über $31,9 \mathrm{mmHg}$ nach 2 Stunden auf $31,0 \mathrm{mmHg}$ nach 4 Stunden ( $\triangleright$ Abb. 5). Es traten damit trotz minimaler Tendenz zu Druckverlusten keine signifikanten oder klinisch relevanten Druckverluste auf.

\section{Diskussion}

\section{Hauptergebnisse}

Das in der Stichprobe abgebildete Geschlechterverhältnis sowie die Verteilungen von phlebolymphostatischer Insuffizienz und Adipositas decken sich mit den Ergebnissen vorheriger Analysen zu Komorbiditäten der Lymphödemerkrankungen [13].

Die Materialeigenschaften, insbesondere die hohe Stiffness, von MAK weisen auf deren therapeutische Effektivität hin. Auch andere Autoren schätzen anhand der aktuell verfügbaren Datenlage die klinische Effektivität vergleichbar gut ein [14]. Hinsichtlich der protokollierten Druckwerte erreichen MAK in Ruhe im Liegen wie im Stehen effektive Druckwerte im Vergleich mit den durch die deutschen Kompressionsklassen vorgegebenen Druckbereichen. Der Static-Stiffness-Index ist bei MAK deutlich höher als bei den vorliegenden FS. Die maximalen Arbeitsdruckwerte und der SSI der MAK bewegen sich tendenziell bereits in Richtung Kurzzugbinden. Dies ist ein wichtiger Faktor für die Behandlungseffektivität hinsichtlich Ödemvolumen und Fibrosierung [15-19].

Die Anwendung von MAK ist dabei auch ohne Nachjustieren sehr druckstabil. Im Gegensatz zu Kurzzugbinden, die über die Tragezeit Druck einbüßen, können MAK den Druck über längere Zeit stabil ausüben. Mosti und Partsch belegten dies über eine deutlich längere Tragezeit und in Selbstanlage mit Nachjustieren; auch in der hier vorliegenden Arbeit kann dies, allerdings ohne Nachjustieren, über 

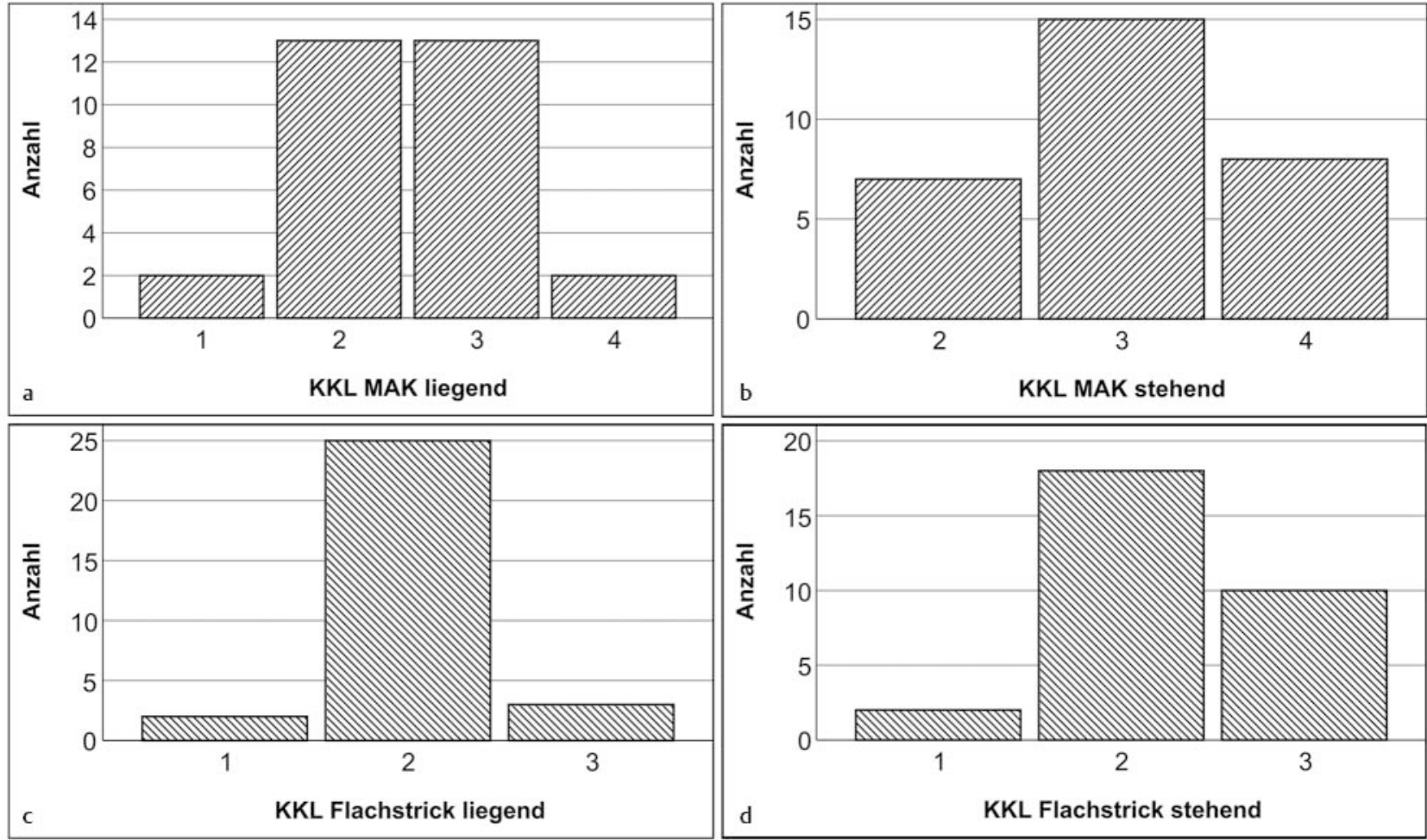

- Abb. 2 Zuordnung der Liegend- und Stehenddruckwerte zu Kompressionsklassen.

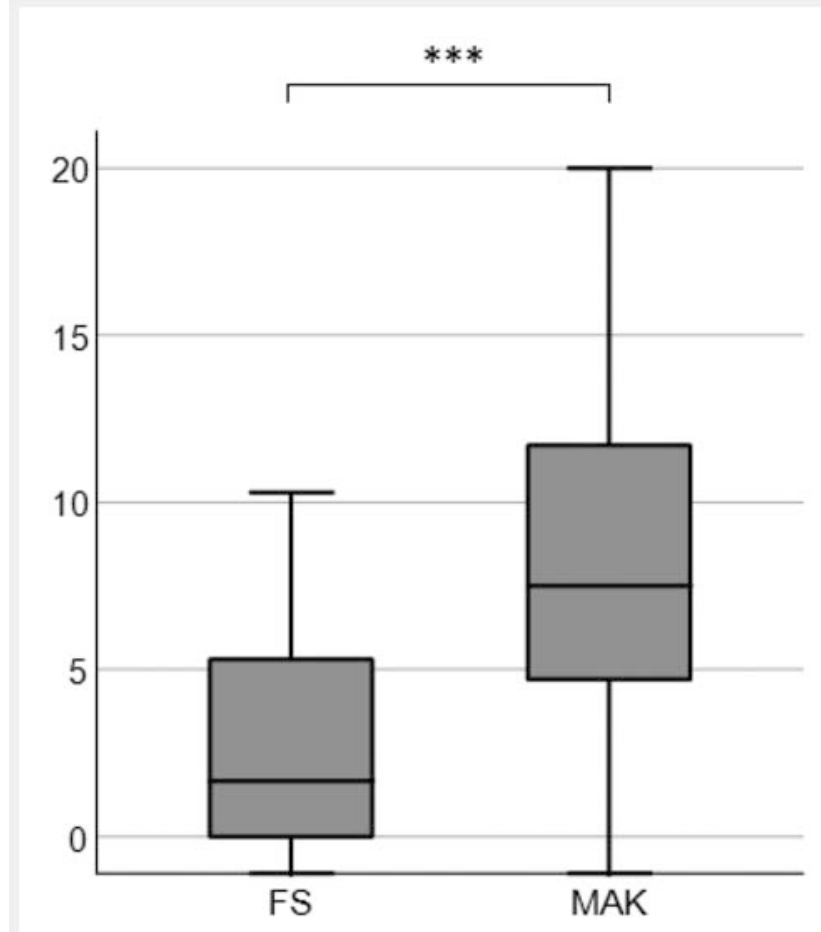

Abb. 3 Static-Stiffness-Index im Vergleich $(p<0,001)$.

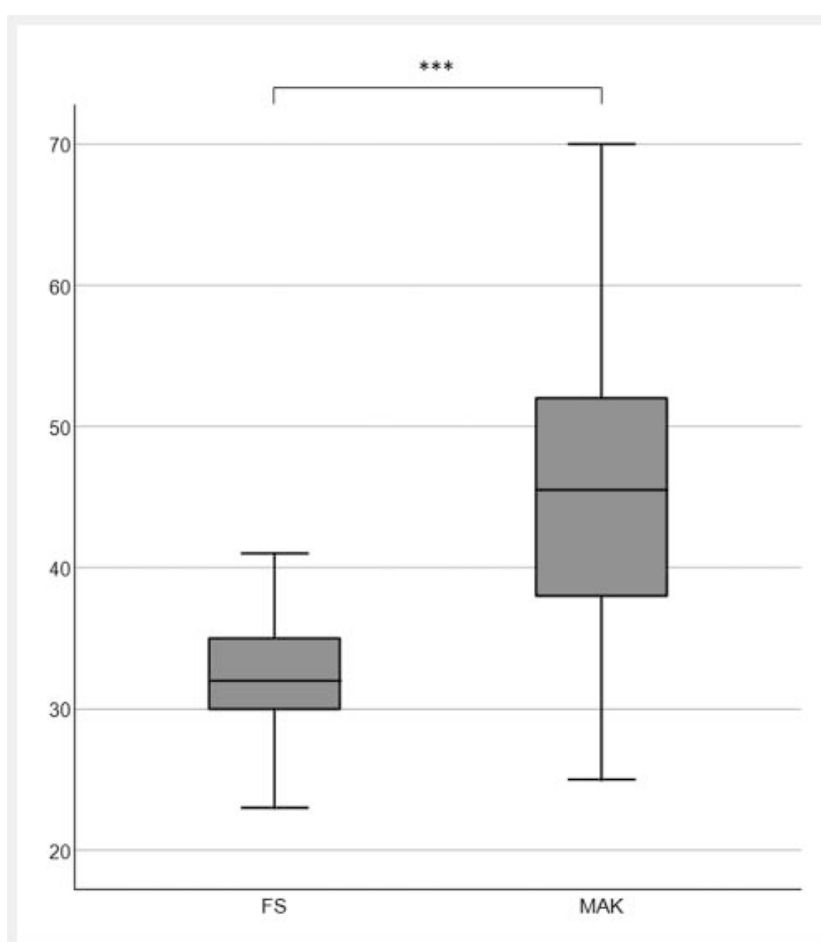

Abb. 4 Maximaler Arbeitsdruck im Vergleich (Boxplots; y-Achse: $\mathrm{mmHg} ; \mathrm{p}<0,001)$. 


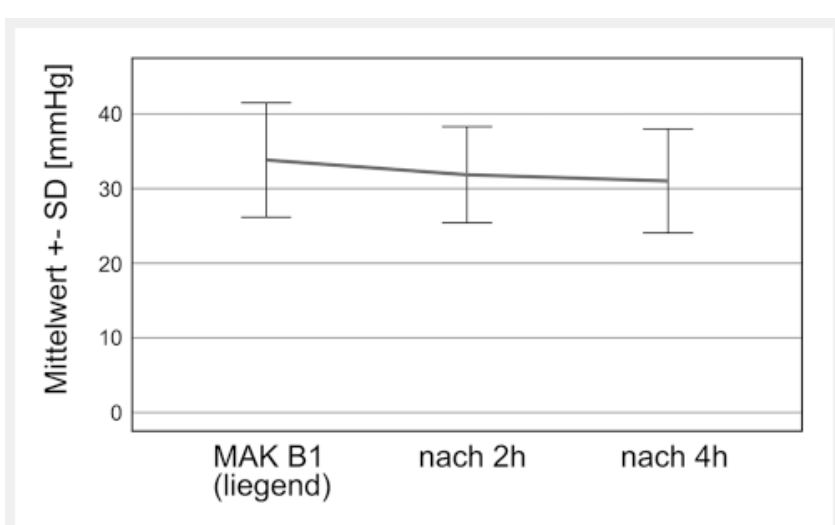

- Abb. 5 Druckmessung Ruhedruck B1 (MAK) im Zeitverlauf nach 2 und 4 Stunden.

4 Stunden gezeigt werden [3, 18]. Dies ist insofern bemerkenswert, als bekanntermaßen die Kompressionswirkung nicht nachjustierbarer Kompressionssysteme wie der Kompressionsbandagierung mit Kurzzugbinden weniger lange anhält [19].

Um valide Ergebnisse bezüglich der Effektivität der Selbstanlage zu erreichen, wurde das Protokoll so gestaltet, dass die Probanden zum Messzeitpunkt 3 wiederholte Messungen erhielten, vor denen jeweils eine Neuanlage der MAK durch die Probanden selbst erfolgte.

Es ist verständlich, dass in diesem experimentellen Setting bei chronischen Ödemen in der Erhaltungsphase auch innerhalb von 4 Stunden Beobachtungszeit nur geringe Volumeneffekte zu erwarten bzw. zu beobachten sein können, die zudem durch zirkadiane und mobilisierungsabhängige Faktoren beeinflusst sind. Bezüglich des Kompressionsdrucks zeigt sich durch die beobachteten Werte, dass in Selbstanlage mit MAK nicht nur kurzfristig effektive Druckwerte und SSI, sondern auch druckstabile Anlagen erreicht werden können. Dies ist natürlich besonders wichtig angesichts der Tatsache, dass die Tragedauer der Kompressionstherapie beim Lymphödem viele Stunden täglich, in der Regel von morgens bis abends, indiziert ist.

Die gemessenen Druckwerte sind durch Selbstanlage der Kompression seitens der Probanden nach sorgfältiger Einweisung in die Handhabung erreicht worden. Die Selbstanlage könnte dabei auch Vorteile hinsichtlich der Therapietreue bringen, im Sinne einer verbesserten Selbstwirksamkeitserwartung. Dies muss insbesondere vor dem Hintergrund verstanden werden, dass sowohl das Anziehen als auch das Ausziehen der MAK viel einfacher empfunden wird als bei Flachstrick-Kompressionsstrümpfen, auch wenn der Tragekomfort nicht als besser empfunden wird [7].

Nebenwirkungen durch Anwendungsfehler traten nicht auf. Die Einweisung in die Handhabung der MAK sollte sorgfältig gemäß den Herstellervorgaben erfolgen und ist von besonderer Bedeutung. 73 \% der Selbstanlagen von MAK zeigten RAL-konforme Druckgradienten. Der mittlere Druckgradient der MAK unterschied sich nicht signifikant von der Situation bei den maßangefertigten und passformgeprüften FS-Strümpfen. Zudem traten auf der FS-Seite in 17\% der Messungen negative Druckgradienten auf, was die Aussagekraft des gemessenen Druckgradienten hinsichtlich der Anwendungssicherheit limitiert. Die Therapiesicherheit mit MAK in Selbstanwendung sollte weiter untersucht werden und ist auch ein Untersuchungsziel fortführender Untersuchungen [7].

\section{Limitationen}

Mögliche systematische Fehler betreffen vor allem das Studiendesign und die Messtechnik. Da die Rekrutierung der Probanden in einer lymphologischen Spezialambulanz erfolgte und unter den Teilnehmern viele Fälle mit langjährig bestehenden Ödemen waren, könnten hier Einflussfaktoren durch langjährig bestehende Gewebeveränderungen oder durch Therapien bestehen. Systematische Messfehler sind ebenfalls denkbar. Es wurde versucht, diese durch die Seitenrandomisierung von Intervention und Kontrolle zu reduzieren.

Die Druckmessungen auf der FS-Seite wurden nicht im Zeitverlauf untersucht und gegen MAK verglichen, da einerseits von konstanten Materialeigenschaften des RAL-konformen Kompressionsstrumpfes auszugehen ist und andererseits die Ziele der Druckmessungen die Feasibility und Kompressionseigenschaften der Selbstanlage der MAK waren. Eine vergleichende Untersuchung von MAK und FS wurde zudem in der beschriebenen RCT vorgenommen [7]. Dennoch könnten durch dieses Studiendesign Gewebeveränderungen unter der Kompression unentdeckt geblieben sein.

Von den Probanden wiesen $10 \%$ (3/30) auch Vorfußödeme auf. Da die Perometrie nur von Knöchel bis Tibiakante durchgeführt wurde, können die Volumenänderungen nicht mit exakten Daten darstellt werden. Klinisch traten jedoch keine Änderungen der Ödembefunde unter MAK zutage.

\section{Zusammenfassung und Ausblick}

Die durchgeführten In-vivo-Druckmessungen zeigen, dass MAK von Patienten mit therapeutisch effektiven Kompressionsdruckwerten selbst angelegt werden können. Die Anwendung von MAK ist auch ohne Nachjustieren druckstabil. Die Anwendung als Selbstanlage kann als therapeutisch wirksam eingestuft werden. Eine gute Einweisung der Patienten ist jedoch bedeutsam. Die gemessenen Druckwerte sind eher im Bereich über der (deutschen) Kompressionsklasse II einzuordnen. Die Stiffness weist tendenziell eher Kurzzugbinden-ähnliche Eigenschaften auf. Weitere Studien sollten therapeutische Optionen mit MAK in den verschiedenen Phasen der KPE und mit Differenzierung verschiedener lymphostatischer Ödeme untersuchen.

\section{Interessenkonflikt}

Die Firma Lohmann \& Rauscher GmbH \& Co. KG (Westerwaldstraße 4, D-56579 Rengsdorf) unterstützte die Studie mit Drittmitteln und stellte das Kompressionsmaterial zur Verfügung. 
Literatur

[1] AWMF. S2k Leitlinie Diagnostik und Therapie der Lymphödeme. AWMF Reg.-Nr. 058-001. https://www.awmf.org/uploads/tx_szleitlinien/ 058-001I_S2k_Diagnostik_und_Therapie_der_Lymphoedeme_ 2019-07.pdf (Zugriff 17.09.2019)

[2] Damstra R], Partsch H. Prospective, randomized, controlled trial comparing the effectiveness of adjustable compression Velcro wraps versus inelastic multicomponent compression bandages in the initial treatment of leg lymphedema. J Vasc Surg Venous Lymphat Disord 2013; 1: 13-19

[3] Mosti G, Cavezzi A, Partsch H et al. Adjustable Velcro Compression Devices are More Effective than Inelastic Bandages in Reducing Venous Edema in the Initial Treatment Phase: A Randomized Controlled Trial. Eur J Vasc Endovasc Surg 2015; 50 (3): 368-374

[4] Reich-Schupke S, Protz K, Dissemond J et al. Neue Entwicklungen in der phlebologischen Kompressionstherapie. Dtsch Med Wochenschr 2017; 142: 679-686

[5] Ehmann S, Whitaker J, Hampton S et al. Multinational, pilot audit of a Velcro adjustable compression wrap system for venous and lymphatic conditions. J Wound Care 2016; 25 (9): 513-520

[6] Stather PW, Petty C, Howard AQ. Review of adjustable velcro wrap devices for venous ulceration. Int Wound J 2019; 16: 903-908

[7] Reißhauer A, Kornappel S, Tsatrafilis E et al. Medizinische Adaptive Kompressionssysteme sind Flachstrick-Kompressionsstrümpfen in der KPE Phase II nicht unterlegen. Phys Med Rehab Kuror 2020; ePub ahead of print doi:10.1055/a-1097-7675

[8] Dissemond J, Protz K, Moelleken M et al. Kompressionstherapie bei Patienten mit Ulcus cruris -welche Kosten entstehen wirklich? Dtsch Med Wochenschr 2019; 144: e94-e101

[9] AWMF. S2k Leitlinie Medizinische Kompressionstherapie der Extremitäten mit Medizinischem Kompressionsstrumpf (MKS), Phlebologischem Kompressionsverband (PKV) und Medizinischen adaptiven Kompressionssystemen (MAK). AWMF-Reg.-Nr. 037/005. https://www.awmf.org/uploads/ tx_szleitlinien/037-005I_S3k_Medizinische-Kompressionstherapie-MKSPKV_2019-05.pdf (Zugriff: 17.09.2019)
[10] RAL Deutsches Institut für Gütesicherung uns Kennzeichnung e. V. Medizinische. Kompressionsstrümpfe Gütesicherung RAL-GZ387/1. 2008. Sankt Augustin. https://www.gzg-kompressionsstruempfe.de/uploads/ media/RAL-GZ_387_1_Ausgabe_01.08.pdf (Zugriff am 08.11.2019)

[11] Partsch H, Clark M, Bassez S et al. Measurement of Lower Leg Compression In Vivo: Recommendations for the Performance of Measurements of Interface Pressure and Stiffness. Dermatol Surg 2006; 32: 224-233

[12] Partsch H, Mosti G. Comparison of three portable instruments to measure compression pressure. Int Angiol 2010; 29 (5): 426-430

[13] Reißhauer A, Schuester L, Kling A et al. Prävalenz von Komorbiditäten be Lymphödemerkrankungen. Phys Med Rehab Kuror 2019; 29 (5): 282289

[14] Dissemond J, Kröger K, Stücker M. Evidenz der Kompressionstherapie unter besonderer Berücksichtigung der medizinischen adaptiven Kompressionssysteme. Hautarzt 2020; 71: 301-308

[15] Blecken SR, Villavicencio JL, Kao TC. Comparison of elastic versus nonelastic compression in bilateral venous ulcers: a randomized trial. J Vasc Surg 2005; 42 (6): 1150-1155

[16] Callam M], Haiart D, Farouk $M$ et al. Effect of time and posture on pressure profiles obtained by three different types of compression. Phlebology 1991; 6: 79-84

[17] Partsch H, Menzinger G, Mostbeck A. Inelastic leg compression is more effective to reduce deep venous refluxes than elastic bandages. Dermatol Surg 1999; 25 (9): 695-700

[18] Mosti G, Partsch H. Druckmessungen unter Klettverschluss-Kompression. Vasomed 2017; 29: 212-216

[19] Protz K, Heyer K, Verheyen-Cronau I et al. Loss of interface pressure in various compression bandage systems over seven days. Dermatology 2014; 229 (4): 343-352 